\title{
Self-Assembling Behavior in Decane Solution of Potential Wax Crystal Nucleators Based on Poly(co-olefins)
}

\author{
Dietmar Schwahn and Dieter Richter* \\ Institute of Solid State Research, Research Center, J ülich, 52425 J ülich, Germany
}

\section{Pamela J . Wright, Charles Symon, and Lewis J . Fetters ${ }^{\dagger}$}

Exxon Mobil Research and Engineering Corporation, Corporate Strategic Research Laboratories, Annandale, New J ersey 08801

\author{
Min Lin \\ Center for Neutron Research, National Institute of Science and Technology, \\ Gaithersburg, Maryland 20899-8562
}

Received J uly 9, 2001; Revised Manuscript Received November 6, 2001

\begin{abstract}
The control of the precipitation and gelation of long chain paraffins from oil remains an enduring technol ogical challenge regarding the processing and recovery of refined fuels and waxy crudes. Wax crystal modifiers based on polyethylene-poly(ethylene-propylene) (PE-PEP) diblock copolymers function as efficient nucleators for wax crystals in middle distillate fuels. These diblock polymers selfassemble in oil to form expansive platelike aggregates consisting of a PE core cloaked behind the amorphous PEP brush layer. The PE core thus promotes nucleation of solubilized long chain alkanes. Additional candidate structures for wax crystal nucleators include linear and star copolyol efins where the composition variation signals the alteration between crystalline and amorphous segments. This study focuses upon the self-assembling behavior in solution of these materials. The characteristics of the single chains and the aggregates formed at lower temperatures were determined via small-angle neutron scattering. Both plates and needlelike structures were found. The placement of the amorphous and crystalline blocks in the arms of the star shaped polymers was found to influence the architecture of the self-assembled micelles. As a point of comparison a commercial copolymer of ethylene-vinyl acetate (EVA) was also investigated. The EVA material was found to be relatively "undiscipl ined" in comparison to the structurally more uniform anionically prepared counterparts.
\end{abstract}

\section{Introduction}

Recently, we have reported on the structure of polymer aggregates formed from polyethylene-poly(ethylene-propylene) diblock copolymers (PE-PEP) in decane. ${ }^{1-3}$ Such diblocks self-assemble to form hairy platelet structures, where the perpendicular dimensions are controlled by molecular weight and composition ratios. Structural information was obtained by small angle neutron scattering (SANS) experiments which led to a structural model, where the diblocks form core PE plates with a thickness range between 20 and $100 \AA$ surrounded on both sides by a PEP polymer brush, where the hairs reach out to about $200 \AA$. Such diblock aggregates have turned out to be extremely successful as templates for wax crystal nucleation, suppressing waxing in diesel fuels. ${ }^{3}$ SANS experiments have shown, that nucleation occurs at the crystalline core surfaces of the PE-PEP platel et structures thus suppressing the growth of large wax crystals.

Here, we present a structural study on the aggregation behavior of more general copolymers containing crystallizable and amorphous chain sections. We vary both the chain topography as well as the block copolymer architecture. Chain topography is investigated in comparing the aggregation behavior of the linear ethylene-butene random copolymer PEB-11 and the ta-

* Corresponding author.

† Present address: Department of Chemical Engineering, Cornell University, Ithaca, NY 14850. pered ethylene-propylene copolymer t-PE-PEP in decane as a solvent. The influence of copolymer architecture is studied on two different star polymers built from PE-PEP diblock copolymers. In one case, the crystallizable chain sections are placed at the rim of the star, while in the other case they are centered at the core. Finally, we compare the aggregation properties of the technical ethylene-vinyl acetate polymer EVA which is used commercially for wax crystal modification. Compared to this technical polymer, the other systems are well-defined, e.g., with respect to their molecular weight compositions and molecular weight distributions.

\section{Experimental Section}

A. Samples. The t-PE-PEP and the PEB-11 were prepared via anionic polymerization in cyclohexane using tert-butyllithium as the initiator. ${ }^{4}$ The "tapered" polymer (t-PE-PEP) was synthesized by the simultaneous presence of butadiene $(0.40)$ and isoprene $(0.60)$ in the reaction solution, where the numbers define the mole fraction of each monomer. The tapered composition of $t-P E-P E P$ results from the relative values of the four chain propagation rate constants which combine to favor the inclusion of butadiene relative to isoprene. ${ }^{4}$ This leads (after hydrogenation) to a high ethylene content segment followed in turn by the gradual enhancement of the alternating ethylene-propylene structure. The molecular weight after hydrogenation was $M_{w}=6.25 \mathrm{~K}$ with $\mathrm{M}_{w} / \mathrm{M}_{n}$ $=1.02$. Molecular weights were measured via size exclusion chromatography (SEC) coupled with on-line light scattering. The PEB-11 polymer is virtually a random poly(ethylene butene) copolymer. It originates from the triethylamine modified anionic polymerization of 1,3-butadiene, where random 
Table 1. Molecular Characteristics of PEB-11a

\begin{tabular}{|c|c|c|c|c|c|}
\hline $\begin{array}{l}\text { triads } \\
\text { and } \\
\text { diads }\end{array}$ & $\begin{array}{c}\mathrm{mol} \% \\
\mathrm{C}_{4}\end{array}$ & $\mathrm{M}_{\mathrm{w}}(\mathrm{g} / \mathrm{mol})$ & MWD & $\begin{array}{c}\text { av DP } \\
\mathrm{C}_{2} \text { and } \mathrm{C}_{4}\end{array}$ & $\begin{array}{l}\text { branches } \\
\text { per } 100 \\
\text { backbone } \\
\text { carbons }\end{array}$ \\
\hline $\begin{array}{l}\text { triads } \\
\text { diads }\end{array}$ & $\begin{array}{l}21.9 \\
\text { EEE } \\
0.46 \\
\text { EE } \\
0.62\end{array}$ & $\begin{array}{l}6.4 K \\
E E B+B E E \\
0.32 \\
E B+B E \\
0.34\end{array}$ & $\begin{array}{l}<1.01 \\
\text { EBE } \\
0.13 \\
\text { BB } \\
0.045\end{array}$ & $\begin{array}{c}118.2 \quad 33.2 \\
E B B+B B E \\
0.09 \\
r_{1} r_{2} \\
1\end{array}$ & \begin{tabular}{lr}
\multicolumn{2}{c}{11.0} \\
BEB & BBB \\
0 & 0 \\
cluster index & \multicolumn{2}{c}{10.5}
\end{tabular} \\
\hline
\end{tabular}

a Definitions of the terms in Table 1: $C_{4}=$ butene; $M_{n}=$ number average molecular weight; $\mathrm{MWD}=$ molecular weight distribution; $\mathrm{C}_{2}=$ ethylene. $\mathrm{EEE}$ and $\mathrm{EE}$ percentage of ethylene triads and diads. $B$ denotes butene. $r_{1} r_{2}=$ the product of the reactivity ratios where convention dictates that $r_{1}$ refers to the majority monomer (in this case ethylene). The cluster index is a measure of randomness; 10 is perfectly random. The PEB polymers cannot be perfectly random since $B E B$ triads are not possible.

Table 2. Molecular Characteristics of the Poly(co-olefin) Starsa

\begin{tabular}{cclcc}
\hline sample & arm $\mathrm{M}_{\mathrm{w}}$ & ${\text { star } \mathrm{M}_{\mathrm{w}}}$ & $\mathrm{f}$ & $\mathrm{M}_{\mathrm{w}} / \mathrm{M}_{\mathrm{n}}$ \\
\hline PE-PEP (DVB) star & 6.5 & $205 \mathrm{~K}$ & 31.5 & 1.12 \\
PEP-PE (DVB) star & 6.5 & $206 \mathrm{~K}$ & 31.7 & 1.11
\end{tabular}

a Definitions of the terms in Table 2: $M_{n}=$ number average molecular weight; $\mathrm{M}_{\mathrm{w}}=$ weight average molecular weight; $\mathrm{f}=\mathrm{star}$ functionality;

placements of the 1,4- and 1,2-units occur. The latter lead to ethyl side branches on an otherwise linear PE following hydrogenation. The ethyl side branch content is about 11 per 100 carbon atoms in the main chain as determined by H NMR of the parent polydiene and ${ }^{13} \mathrm{C}$ NMR on the poly(co-olefins). The characterization data are given in Table 1 . The EVA copolymer (provided by Infinium, Ltd.) is classified as a nucleating agent and contains 9 mol \%/VA. This copolymer represents a long existing commercial middle distillate fuel additive.

Two star polymers were constructed in cyclohexane by first preparing, via anionic polymerization, a hydrogenous diene based block copolymer followed by the addition of divinylbenzene which served to link the independent arms into the star structure. ${ }^{5}$ The polyisoprene and polybutadiene segments contained the expected high 1,4 (ca. 90\%) microstructure. This led to the formation of the amorphous alternating poly(ethylene-propylene) (PEP) connected to the semicrystalline polyethylene block. The polymerization sequences were such as to lead to a star shaped copolymer with an amorphous corona and semicrystalline core and vice versa. The star with the crystallizable outer rim is dubbed the PE-PEP(DVB) star while that with the amorphous rim is the PEP-PE (DVB) star.

All samples examined in this work were fully protonated and were dissolved in $n$-decane $\left(d_{22}\right)$ in order to achieve the highest possible neutron scattering contrast. The saturation of the two linear polydienes was done using the palladium barium sulfate catalyst. The hydrogenation of the star polymers was done in several repetitive runs using first the palladium/barium sulfate system followed by the soluble Wilkenson catalyst. Two to three runs were needed for complete saturation. The molecular weight measurements were done using an on-line light scattering system (Viskotek) coupled to a size exclusion chromatograph (Waters). The latter was equipped with six linear Phenomonex columns with degassed THF as the carrier solvent at $35^{\circ} \mathrm{C}$. Both the parent polydiene diblocks and the star copolymers were evaluated prior to hydrogenation. From the measured diene compositions and the diblock molecular weights the arm segment molecular weights were $P E P=5 K$ and $P E=1.5 K$. Table 2 contains the star characterization data. The star molecular weight data are from SANS measurements. The functionality data show that the stars in solution are approximate hard spheres. ${ }^{8}$ The amount of unlinked arm in the stars ranged from 2 to $3 \mathrm{wt} \%$.

B. SANS Experiments. The scattering data for the linear copolymers were accumulated at the small angle neutron

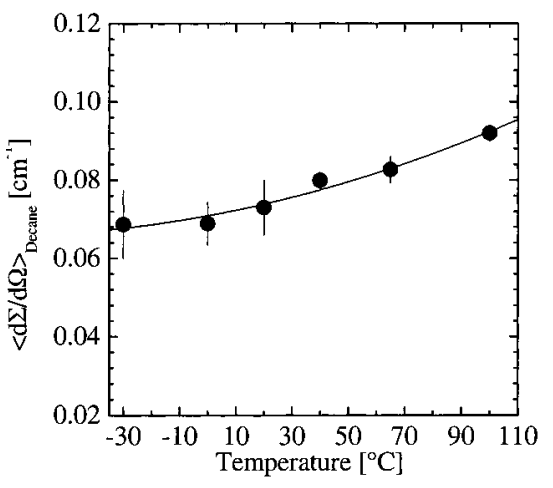

Figure 1. Absolute cross section for $\mathrm{d}_{22}$-n-decane as a function of temperature. The solid line is a result of a polynomial fit.

scattering (SANS) facilities of the Laboratoire Léon Brillouin in Saclay, France, and at the FRJ -2 research reactor in J ülich, Germany. The $\mathrm{Q}$ ranges were within $0.0045<\mathrm{Q}<0.09 \AA^{-1}$ and $0.0015<Q<0.14 \AA^{-1}$ in Saclay and J ülich, respectively. The momentum transfer is defined as $Q=(4 \pi / \lambda) \sin (\theta / 2)$ with $\lambda=$ neutron wavel ength and $\theta=$ scattering angle. The SANS measurements on the stars were done at the CNRS at NIST, Gaithersburg, MD, using the NG-7 (30 m) instrument. The data were corrected for background scattering measured with an empty cell. The Saclay and J ülich data were calibrated to absolute units by a water and a Lupolen standard, respectively. At NIST a secondary standard from $\mathrm{SiO}_{2}$ powder was used. The absolute cross section of the decane- $d_{22}$ solvent was measured at all different instruments. The obtained absolute values agreed within $10-20 \%$. We later on normalized all spectra to the respective decane cross section as depicted in Figure 1. The decane cross sections thereby correspond to a water cross section of $\langle\mathrm{d} \Sigma / \mathrm{d} \Omega\rangle=0.96 \mathrm{~cm}^{-1}$ measured with a $0.1 \mathrm{~cm}$ thick $\mathrm{H}_{2} \mathrm{O}$ sample with $7 \AA$ neutrons. ${ }^{9}$ The scattering intensity of the decane- $d_{22}$ solvent was investigated covering the temperature range of the experiment and subtracted from the respective SANS data.

In the following, we first analyze the scattering from single polymers and then from the polymer aggregates. The following strategy was followed:

(i) At high temperature, where self-assembly is absent, we studied the single chain properties. Measurements at different volume fractions led to the molecular weight, the second virial coefficient and the radius of gyration.

(ii) The aggregation behavior of the polymers was studied as a function of temperature covering a range of -30 to 100 ${ }^{\circ} \mathrm{C}$. Two different pol ymer volume fractions $\Phi_{\mathrm{pol}}=1 \%$ and $\Phi_{\mathrm{pol}}$ $=2 \%$ were investigated, and $2 \%$ was the standard concentration for all the previous experiments. ${ }^{1,3}$ The $1 \%$ system was the highest one at which the structure factor of the prototype 1.5/5 PE - PE P diblock copolymer did not show any concentration effects. ${ }^{3}$

\section{Experimental Results and Analysis of the SANS Data}

A. Single Chain Properties. The results of the polymer solutions at $100{ }^{\circ} \mathrm{C}$ are shown in Figure 2 for $\Phi_{\text {pol }}=2,1,0.5$, and $0.2 \%$ volume fractions of the PEB11 and t-PE-PEP materials. The data sets have been divided by their corresponding polymer volume fraction, respectively. As may be seen the data do not collapse to a single curve. This indicates the influence of the second virial coefficient. In the following step the data were analyzed in terms of the Zimm approximation. ${ }^{6}$

$$
\mathrm{S}(\mathrm{Q})^{-1} \equiv\left[\frac{\mathrm{d} \Sigma}{\mathrm{d} \Omega}(\mathrm{Q}) \mathrm{K}\right]^{-1}=(\Phi \mathrm{V})^{-1}+2 \mathrm{~A}_{2}+\mathrm{LQ}^{2}
$$

Here $\mathrm{S}(\mathrm{Q})$ denotes the structure factor, $\mathrm{K}=\mathrm{N}_{\mathrm{A}} / \Delta \rho^{2}$ the inverse contrast factor, $\mathrm{V}$ the molar volume, $\mathrm{A}_{2}$ the 


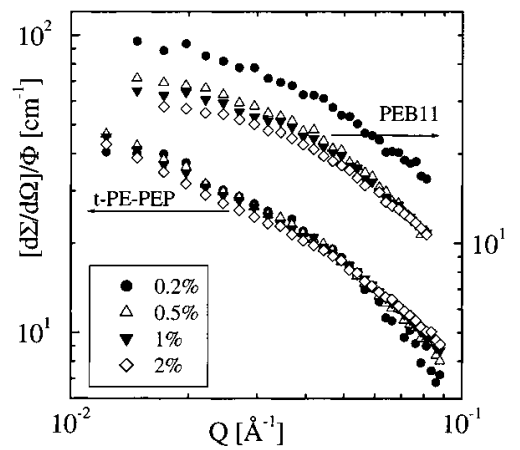

Figure 2. Experimental cross sections from PEB-1land t-PE-PEP at $96{ }^{\circ} \mathrm{C}$ for different volume fractions after a division by the polymer volume fraction.

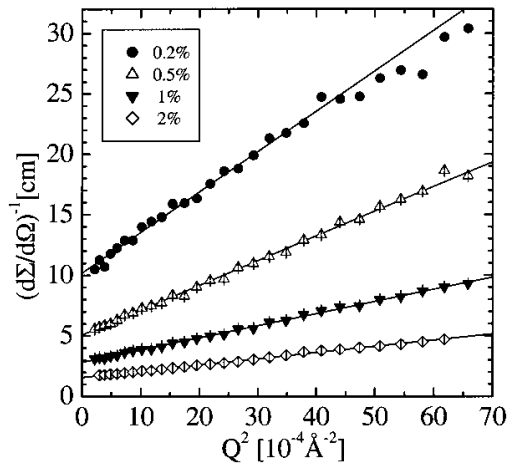

Figure 3. Zimm representation of the PEB-11 data taken at $96{ }^{\circ} \mathrm{C}$ for different polymer volume fractions. The solid lines represent the expected $\mathrm{Q}^{2}$ power laws.

second virial coefficient, and $\mathrm{L}=\mathrm{R}_{\mathrm{g}}{ }^{2} /(3 \Phi \mathrm{V}) . \mathrm{N}_{\mathrm{A}}$ is the A vogadro number and $\Delta \rho$ the difference of the coherent scattering length densities of the polymer and solvent molecules ( $\rho=\sum \mathrm{b}_{\mathrm{i}} / v$, where $\mathrm{b}_{\mathrm{i}}$ are the scattering lengths and $v$ is the volume of the scattering unit).

The data obtained from PEB-11 are plotted in the Zimm representation in Figure 3. Over the whole concentration range from 0.2 to 2 vol \% we observe a linear dependence on $\mathrm{Q}^{2}$ in agreement with the Zimm approximation of eq 1 . From this plot we extract the "forward" scattering at $\mathrm{Q}=0$ by extrapolation and the slope $\mathrm{L}$ which delivers the radius of gyration of the polymers according to $\left\langle\mathrm{R}_{\mathrm{g}}{ }^{2}\right\rangle=3 \mathrm{~L} \Phi \mathrm{V}$. Following eq 1 , the forward scattering also depends on the second virial coefficient, $A_{2}$. In Figure $4 a, S^{-1}(0)$ is plotted vs $1 / \Phi$ yielding both the molar volume (slope) as well as $A_{2}$ (intersection $1 / \Phi=0$ ). From this plot a second virial coefficient $A_{2}=6.7 \times 10^{-3} \mathrm{~mol} / \mathrm{cm}^{3}$ and a molar vol ume of $\mathrm{V}=7400 \mathrm{~cm}^{3} / \mathrm{mol}$ is obtained. The resulting molecular weight $\mathrm{M}_{\mathrm{w}}=6.4 \mathrm{~K}$ compares favorably with the SE C result of $6.6 \mathrm{~K}$. In Figure $4 \mathrm{~b}$ the corresponding radii of gyration are plotted vs concentration; for all concentrations but $0.2 \%$, we obtain the same value, $R_{g}=42.5$ $\AA$. The same analysis was performed for the tapered sample. In contrast to the PEB-11 solutions the t-PE PEP samples always exhibit an increase of the scattered intensity bel ow $\mathrm{Q}<0.02 \AA^{-1}$. This indicates that even at this high temperature a small fraction of larger objects are present at all volume fractions. A Zimm evaluation of the data for $Q>0.02 \AA^{-1}$ yields the values for molecular volume and the second virial coefficient $\mathrm{V}=6100 \mathrm{~cm}^{3} / \mathrm{mol}$ and $A_{2}=4.9 \times 10^{-3} \mathrm{~mol} / \mathrm{cm}^{3}$. The SANS and the SEC molecular weights differ somewhat (5.4K and $6.25 \mathrm{~K})$. Finally for the radius of gyration a value of $R_{g}=40 \AA$ evolves.

B. Aggregation Behavior of the PEB-11 and the t-PE-PE P Samples: Qualitative Overview. In Figure 5, the SANS macroscopic scattering cross sections $\mathrm{d} \Sigma / \mathrm{d} \Omega$ vs the momentum transfer $\mathrm{Q}$ have been depicted in the double logarithmic scale for both polymers at $-30{ }^{\circ} \mathrm{C}$. The characteristic feature for both samples is the appearance of power laws according to

$$
\frac{\mathrm{d} \Sigma}{\mathrm{d} \Omega}(\mathrm{Q}) \propto \mathrm{Q}^{-\alpha}
$$

with an exponent $\alpha$ characteristic for certain spatial arrangements of the polymer and aggregation structure, respectively. At large $\mathrm{Q}$ in both samples one finds an exponent $\alpha \cong 1$.6. Such an exponent is expected from the excluded volume interaction between the monomers of single chains in a "good" solvent. There the conformation of a polymer chain follows a self-avoiding random walk with a fractal dimension $\mathrm{D}=1 / v$, where $v=3 / 5$ is the Flory exponent.

The increase of the intensity at lower Q is caused by the polymer aggregates. The power laws give qualitative information about their morphology. In the intermediate Q-range, a $\mathrm{Q}^{-1}$ power law is observed, while at low $\mathrm{Q}$ a further increase of the intensity is found which for the tapered polymers leads to another power law with an exponent $\alpha \cong 3$. The exponents $\alpha=1$ observed for the PEB-11 sample over a large $Q$ range and the exponent $\alpha=3$ observed for the tapered sample are indicative for a rodlike 1-dimensional, and a 3-d porous structure, respectively. In general if we consider a fractal object
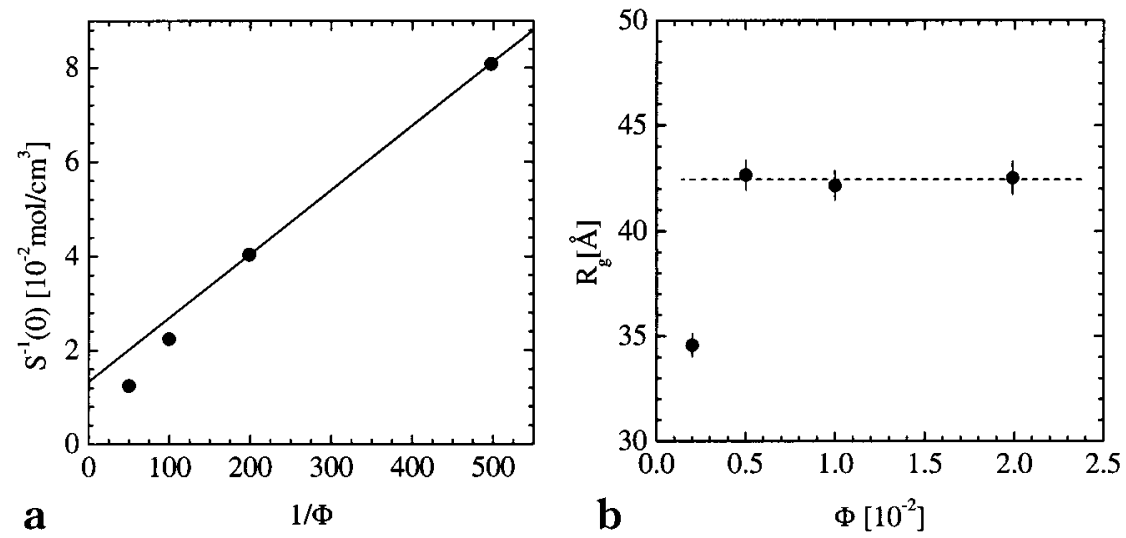

Figure 4. (a) Inverse forward scattering from the PEB-11 polymer at $96{ }^{\circ} \mathrm{C}$ as a function of inverse volume fraction. The solid line represents the theoretical linear relationship. (b) Radius of gyration from PEB-11 as a function of polymer volume fraction. The dashed line serves as a guide to the eyes. 


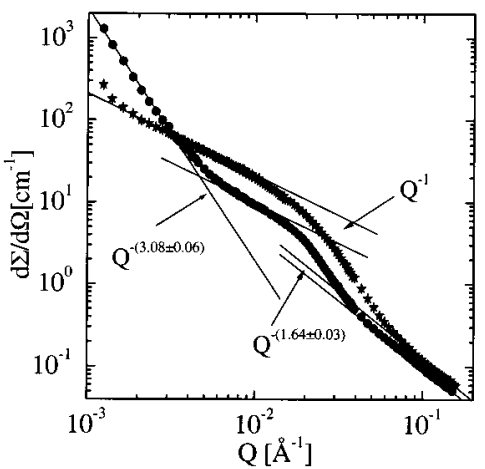

Figure 5. Scattering cross sections at $\Phi_{\text {pol }}=2 \%$ from PE B$11(*)$ and t-PE -PEP (0) in decane at $-30{ }^{\circ} \mathrm{C}$ on a double logarithmic scale. The straight lines indicate power law relations in different $\mathrm{Q}$ ranges.

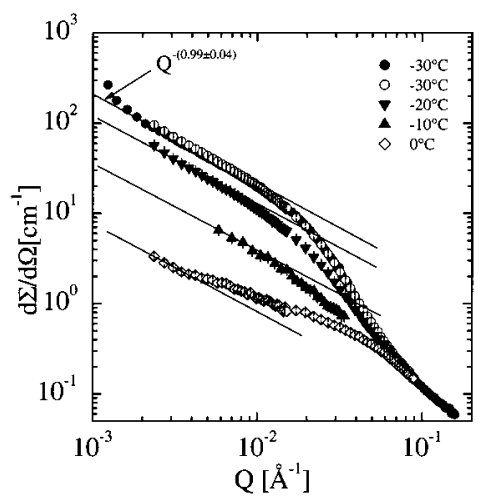

Figure 6. SANS profiles from PEB-11 at different temperatures at $\Phi_{\text {pol }}=2 \%$. The solid lines in the low-Q regime indicate the asymptotic $\mathrm{Q}^{-1}$ power laws for rodlike scattering.

of fractal dimension $D$, i.e., its mass $M$ scales with its size $R$ according to $M \propto R^{D}$, then for the scattering cross section from this object follows: $\left\{d \Sigma / d Q \propto Q^{-D}\right\}(\alpha \equiv$ $D)$. Thus a power law regime in $Q$ with an exponent $D$ $\leq 3$ relates to a fractal object of dimension $D$. In this sense the exponent $\alpha=3$ observed for the tapered copolymer at low $\mathrm{Q}$ indicates that at spatial dimensions $\mathrm{R} \geq 2 \pi / \mathrm{Q}^{*}$, where $\mathrm{Q} *$ is the crossover point to the $\mathrm{Q}^{-3}$ power law, the polymer aggregates resemble 3-d porous objects. In the following sections, we will often argue in terms of power laws and length scales. Thus, the structural information and conclusions presented in this report are based upon the experimentally SANS evaluated behavior of the fractal dimension $\mathrm{D}$ on $\mathrm{Q}$. The underlying theory of fractal structures and the corresponding results in SANS can be found in refs 10 an 11, respectively.

C. Detailed Aggregation Behavior of PE B-11 and t-PE-PE P. We commence with the random copolymer PEB-11. Figure 6 displays a summary of the cross sections measured at different temperatures for $\Phi_{\mathrm{pol}}=$ $2 \%$. The $\Phi_{\text {pol }}=1 \%$ sample shows a similar behavior. With decreasing temperature at low $\mathrm{Q}$ the scattering intensity is rapidly increasing and gradual ly enhances to the scattering pattern of Figure 5 which at low $Q$ is dominated by a very extended range of a $\mathrm{Q}^{-1}$ power law relationship. At high $\mathrm{Q}$ for all temperatures, a tendency toward a $\mathrm{Q}^{-1 / v}$ asymptote with the Flory exponent $v=$ 0.6 is observed (shown in Figure 5). It is interesting to note that the intensity level of this asymptote, if at all, appears to have only a very minor temperature dependence. Thus, though the polymers are aggregating, on a local scale the diffuse scattering from the monomer

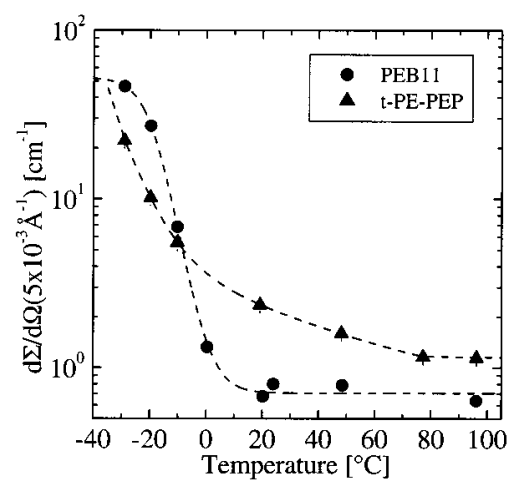

Figure 7. Absolute cross section from the $P E B-11$ and t-PE PEP solutions for $\Phi_{\text {pol }}=2 \%$ at $Q=5 \times 10^{-3} \AA^{-1}$ as a function of temperature. The dashed lines serve as a guide for the eye.

correlations within a swollen chain prevails. The rod structures must be very open exhibiting single chain structures locally.

To better depict the intensity increase with decreasing temperature, Figure 7 displays the measured intensity at $\mathrm{Q}=5 \times 10^{-3} \AA^{-1}$ as a function of temperature. It is seen that the PEB-11 polymer is completely soluble (single chain behavior) at room temperature and higher. Below $0{ }^{\circ} \mathrm{C}$, the polymers gradually commence the selfassembly process into larger objects. Besides the qual itative observation on the general temperature dependence of aggregation, no further quantitative conclusions can be drawn from Figure 7. The size of the objects is not accessible since no saturation of the SANS intensity toward small Q is visible in Figure 6. Thus the length scales of the aggregates need to be larger than several $1000 \AA$ in order to be compatible with the low-Q observations. A comparison of the 1 and $2 \%$ samples shows that other than the proportionality with $\Phi_{\text {pol }}$ no further dependence on the polymer volume fraction is visible.

The extended $\mathrm{Q}^{-1}$ power law found below $\mathrm{Q} \cong 10^{-2}$ $\AA^{-1}$ indicates linear rodlike aggregates. From the bending down of the intensity toward larger $Q$ bel ow the $Q^{-1}$ line the lateral size of rods may be accessed. For 1-dimensional objects the scattering cross section has the general form

$$
\frac{\mathrm{d} \Sigma}{\mathrm{d} \Omega}(\mathrm{Q})=\Phi_{\text {rod }}\left(1-\Phi_{\text {rod }}\right) \pi \mathrm{F}_{\text {rod }} \Delta \rho_{\text {rod }}{ }^{2} \exp \left(-\mathrm{a}^{2} \mathrm{Q}^{2} / 4\right) / \mathrm{Q}
$$

where $F_{\text {rod }}=\pi \mathrm{a}^{2}$ is the area of the rod, a the rod radius, $\Phi_{\text {rod }}$ their volume fraction, and $\Delta \rho_{\text {rod }}$ the difference in scattering length density between the solvent and the rod region. Note that if the rod contains solvent, then the average over the polymer and solvent content has to be taken. If one displays $\ln (\mathrm{Q}[\mathrm{d} \Sigma / \mathrm{d} \Omega])$ vs $\mathrm{Q}^{2}$, then such a 1-d Guinier representation directly reveals the rod radius. Figure 8 presents the low-temperature data for $\Phi_{\mathrm{pol}}=1 \%$ in such a plot. Pronounced linear regimes allowing an evaluation of the lateral rod size are observed. The resulting values for the rod radii and the forward scattering $\left.(\mathrm{d} \Sigma / \mathrm{d} \Omega) \mathrm{Q}\right|_{\mathrm{Q}=0}$ are listed in Table 3 . Similarly al so the data from the $\Phi_{\mathrm{pol}}=2 \%$ solution were evaluated. The corresponding results are also displayed in Table 3.

The aggregation of the tapered polymer commences at a higher temperature than PEB-11. We noted a small amount of large objects present even at $96.2^{\circ} \mathrm{C}$. Scattering data are available down to $-30^{\circ} \mathrm{C}$ and are shown 


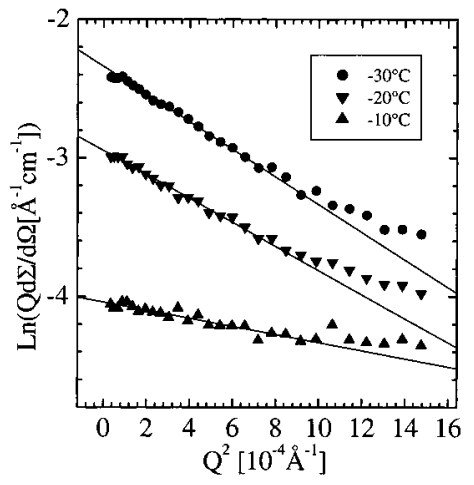

Figure 8. PEB-11 results at $\Phi_{\mathrm{pol}}=1 \%$ in $1-\mathrm{d}$ Guinier presentation.

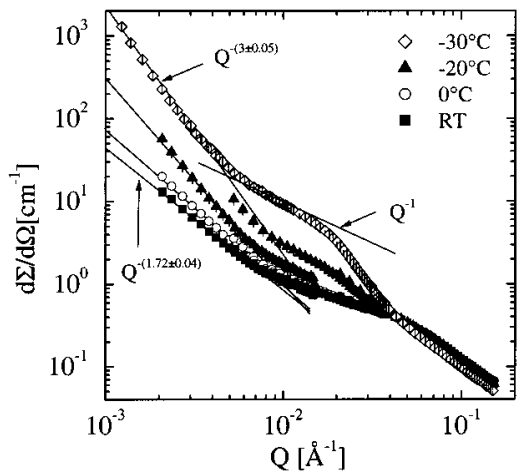

Figure 9. SANS profiles from t-PE-PEP for different temperatures and $\Phi_{\text {pol }}=1 \%$. The various solid lines indicate power law regimes.

Table 3. Rod Parameters and Forward Scattering from the Rodlike Aggregates Formed by the PEB-11 and t-PE-PEP Samples

\begin{tabular}{|c|c|c|c|c|c|}
\hline \multirow[b]{3}{*}{$\begin{array}{c}\text { temp } \\
\left({ }^{\circ} \mathrm{C}\right)\end{array}$} & \multicolumn{4}{|c|}{ polymer } & \multirow[b]{3}{*}{$\begin{array}{l}\text { fractal } \\
\text { expt D }\end{array}$} \\
\hline & \multicolumn{2}{|c|}{ PEB-11 } & \multicolumn{2}{|c|}{ t-PE-PEP } & \\
\hline & a $(\AA)$ & $\begin{array}{c}(\mathrm{d} \Sigma / \mathrm{d} \Omega) \mathrm{Q}_{\mathrm{Q}=0}=0 \\
\left(10^{6} \mathrm{~cm}^{-2}\right)\end{array}$ & $a(\AA)$ & $\begin{array}{c}\left.(\mathrm{d} \Sigma / \mathrm{d} \Omega) \mathrm{Q}\right|_{\mathrm{Q}=0} \\
\left(10^{6} \mathrm{~cm}^{-2}\right)\end{array}$ & \\
\hline \multicolumn{6}{|c|}{$\Phi=1 \%$} \\
\hline $\begin{array}{l}-10 \\
-20 \\
-30\end{array}$ & $\begin{array}{l}42 \pm 2 \\
72 \pm 1 \\
78 \pm 1\end{array}$ & $\begin{array}{l}1.76 \pm 0.02 \\
5.2 \pm 0.1 \\
9.6 \pm 0.1\end{array}$ & $\begin{array}{l}46 \pm 3 \\
97.5 \pm 1\end{array}$ & $\begin{array}{l}1.25 \pm 0.05 \\
6.7 \pm 0.1\end{array}$ & $\begin{array}{l}2.92 \\
2.86\end{array}$ \\
\hline \multicolumn{6}{|c|}{$\Phi=2 \%$} \\
\hline $\begin{array}{l}-10 \\
-20 \\
-30\end{array}$ & $\begin{array}{l}37 \pm 3 \\
82 \pm 2 \\
83 \pm 1\end{array}$ & $\begin{array}{l}0.55 \pm 0.01 \\
12.6 \pm 0.1 \\
23.0 \pm 0.1\end{array}$ & $\begin{array}{l}58 \pm 1.5 \\
82 \pm 5\end{array}$ & $\begin{array}{l}3.4 \pm 0.1 \\
14 \pm 0.2\end{array}$ & $\begin{array}{l}2.47 \\
3.08\end{array}$ \\
\hline
\end{tabular}

in Figure 9 where the results for the polymer volume fractions $\Phi_{\mathrm{pol}}=2 \%$ are displayed. The possibility of analysis for the objects observed at high temperature is limited. The aggregates could represent some small amount of branched structures originating from side reaction of the living chain end with the side chain vinyl double bonds during synthesis. ${ }^{12}$

Again in order to qualitatively visualize the build up of intensity with decreasing temperature, Figure 7 presents the cross section at $Q=5 \times 10^{-3} \AA^{-1}$ as a function of temperature. Due to the presence of a small amount of polymer clusters even at the highest temperature, the observed intensity is higher than the single coil scattering. Other than for PEB-11, the further intensity increases set in al ready above room temperature and continue to grow toward lower temperatures. Thus, while the PEB-11 material only aggregates below $0{ }^{\circ} \mathrm{C}$ the tapered polymer exhibits first signs of aggregation around $50{ }^{\circ} \mathrm{C}$. This behavior displays some similarity to that of the discrete diblocks. ${ }^{3}$

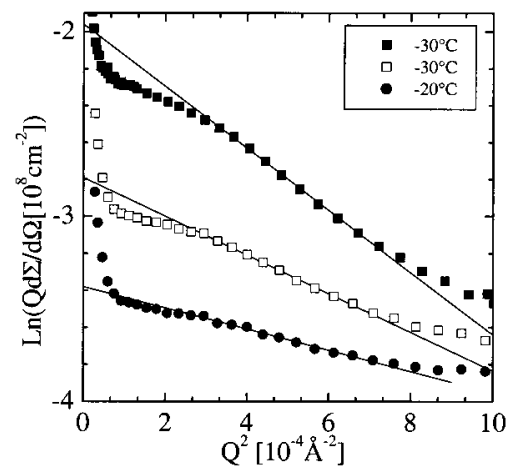

Figure 10. Guinier analysis in the intermediate rod regime for t-PE-PEP at various temperatures $\Phi_{\text {pol }}=2 \%$.

To proceed further, we first notice that also for the t-PE-PEP there is an indication of $\mathrm{Q}^{-1}$ behavior at intermediate Q's due to a rodlike structure (Figure 9). However, other than for PEB-11 this structure is limited to a narrow $\mathrm{Q}$ or length scale regime and only evolves at $\mathrm{T}=-20^{\circ} \mathrm{C}$ and $\mathrm{T}=-30^{\circ} \mathrm{C}$. Figure 10 presents a 1-d Guinier analysis for the $2 \%$ sample following eq 3. The resulting parameters are quoted in Table 3 . In Figure 10 the two different results at $-30^{\circ} \mathrm{C}$ are shown. They originate from a measurement directly after cooling and another after saturation of the intensity after a waiting period of about $3 \mathrm{~h}$. Obviously the rate of aggregateformation is slow and reproduci bility is only achieved after waiting for saturation.

Figure 11 displays the temperature dependent rod radi $i$ and the extrapolated forward scattering from the 1-d Guinier plot for both polymers. The forward scattering for both volume fractions changes linearly with temperature and reaches zero at -9 and $-17^{\circ} \mathrm{C}$ for the PE B-11 and t-PE - PEP, respectively. The rod radius for the PEB-11 material appears to saturate at low temperatures, while this quantity increases steadily with decreasing temperature for the tapered polymer. We note that upon cooling the rod structures form first for the PEB-11 sample; this behavior is opposite to the general aggregation trends displayed in Figure 7 . The tapered material commences to aggregate al ready well above room temperature, while PEB-11 shows signs of aggregation only below $\mathrm{T}=0{ }^{\circ} \mathrm{C}$. Thus, while PEB-11 upon aggregation assumes more or less immediately the rodlike morphology, at higher temperatures the t-PEPEP does not exhibit this rodlike regime.

The scattered intensity from the tapered polymer solutions exhi bit a strong power law toward low Q. The corresponding fractal exponents are plotted in Figure 12 for the $1 \%$ and $2 \%$ solutions vs temperature. For both solutions the fractal exponent increases with increasing temperature. Hence, this increase is more pronounced for the higher polymer volume fraction. At the lowest temperature for both solutions the exponent becomes 3 , indicating the presence of 3-d porous objects.

From these findings we may conclude that polymers with random crystallizable sections have the tendency to aggregate into rodl ike structures. These rods become more pronounced and extended for the PEB-11 material, which contains relatively short PE sections. At the lowest temperature rod structures of roughly $3000 \AA$ length and about $80 \AA$ radius are formed. Furthermore, at the lowest $\mathrm{Q}$ values there is some indication for an intensity turn up indicating branching of the rods.

The structures built by the self-assembly of the tapered polymers are more complex. At low tempera- 

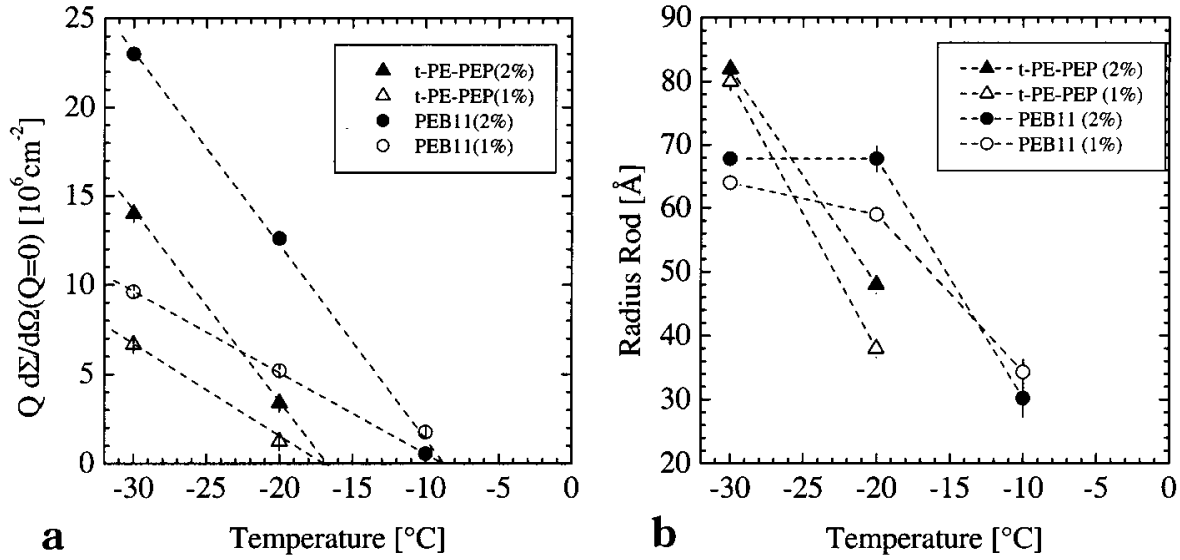

Figure 11. (a) Temperature dependence of the rod forward scattering from the tapered t-PE -PEP and the random copolymer PEB-11 samples. The dashed lines are linear interpolations between corresponding points. Note that the results for the two different vol ume fractions investigated point to a common starting point for rod formation in each case. (b) Temperature-dependent rod radius for the two polymers. The dashed lines are guides to the eye.

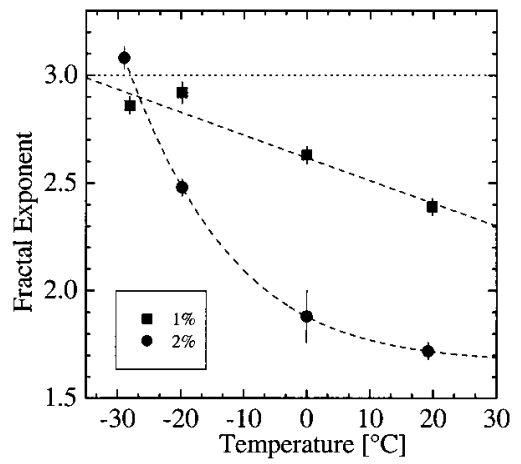

Figure 12. Fractal exponents from the t-PE-PEP solutions in the low-Q regime as a function of temperature. The dashed lines are guides to the eye. The pointed line indicates the $D=$ 3.0 threshold.

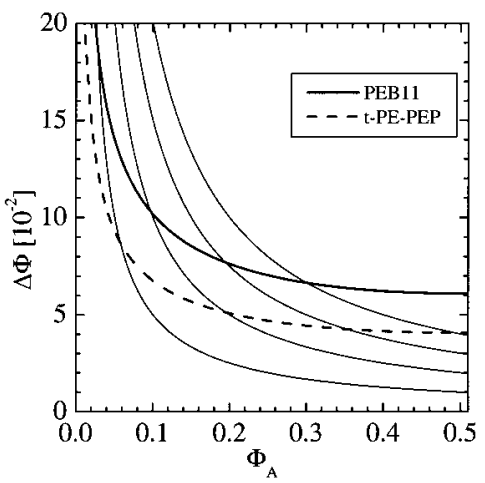

Figure 13. Difference between the aggregated and free polymer volume fractions $\Delta \Phi$ as a function of the volume fraction of aggregated polymer $\Phi_{\text {rod }}$ for the tapered (dashed line) and the random copolymer (solid line). The thin solid lines represent $\Delta \Phi=\left(\Phi-\Phi_{\mathrm{pol}}^{\mathrm{sol}}\right) / \Phi_{\text {rod }}$ (see text) for different $\Phi_{\mathrm{pol}}^{\mathrm{sol}}$ from above $0,0.005,0.01$, and 0.015 .

tures again rods are formed but these rods are much shorter $(\sim 600 \AA)$ and form fractal network structures which at $-30{ }^{\circ} \mathrm{C}$ are of a 3-dimensional nature. Above $-17{ }^{\circ} \mathrm{C}$ rod formation is not visible. The data rather indicate fractal aggregate structures with fractal dimensions below 3.

D. Polymer Density within the Aggregates and Aggregate Volume Fraction. Neutron small angle scattering reveals not only momentum transfer dependent scattering patterns giving information about structural details but also absolute intensities which relate

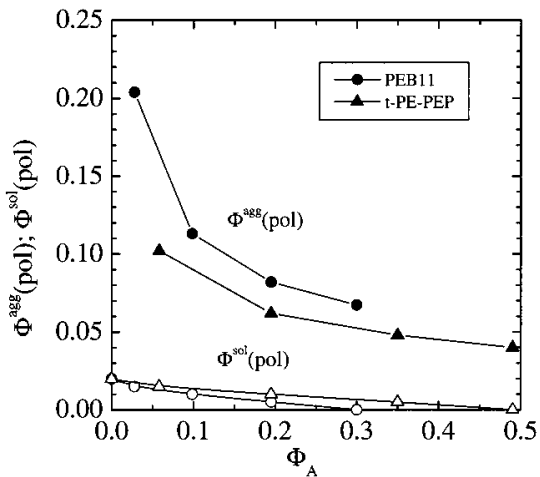

Figure 14. Aggregated polymer volume fractions $\Phi_{\mathrm{pol}}^{\mathrm{agg}}$ and solved volume fractions $\Phi_{\text {pol }}^{\text {sol }}$ for PEB-11 and the tapered polymer as a function of the aggregated fraction $\Phi_{\text {rod }}$.

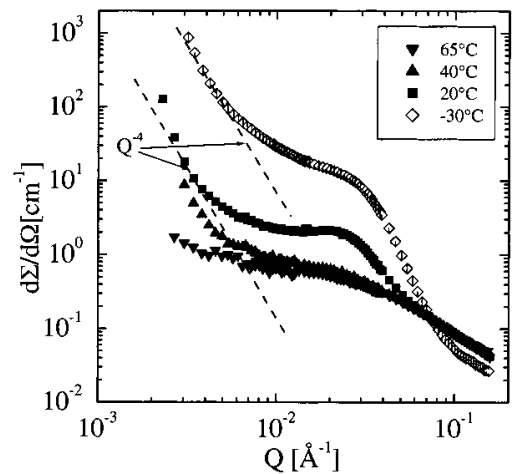

Figure 15. Cross sections observed from EVA at a volume fraction of $\Phi_{\text {pol }}=2 \%$ for different temperatures. The solid lines indicate the asymptotic Porod Iaw $\left(\mathrm{Q}^{-4}\right)$.

to the vol umes of the scattering objects. In our case, we have to face a partition of the dissolved polymers between the self-assembled aggregates and the remaining single chains in sol ution. Therefore, it is not possible to give single valued parameters about the volume fraction of the sample which is occupied by the aggregated polymers and the polymer density within the aggregate structures. However, both quantities are related to each other. On the basis of eq 3 (which is valid for rod-scattering and the assumption that at low temperature the basic el ement of the aggregate structures are rods), we can quantify the relation between aggregate volume fraction and polymer density within the rods. Table 4 contains the necessary definitions: 


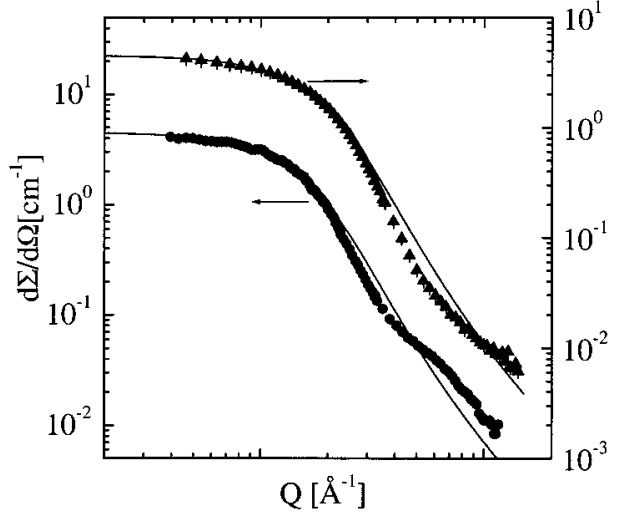

Figure 16. Single star form factors at $\mathrm{T}=71{ }^{\circ} \mathrm{C}$ for the $\mathrm{PE}-$ PEP (DVB) and PEP-PE (DVB) stars at $\Phi=0.024 \%$ in decane solution. The solid line represents a fit with the Gaussian star form factor (eq 7).
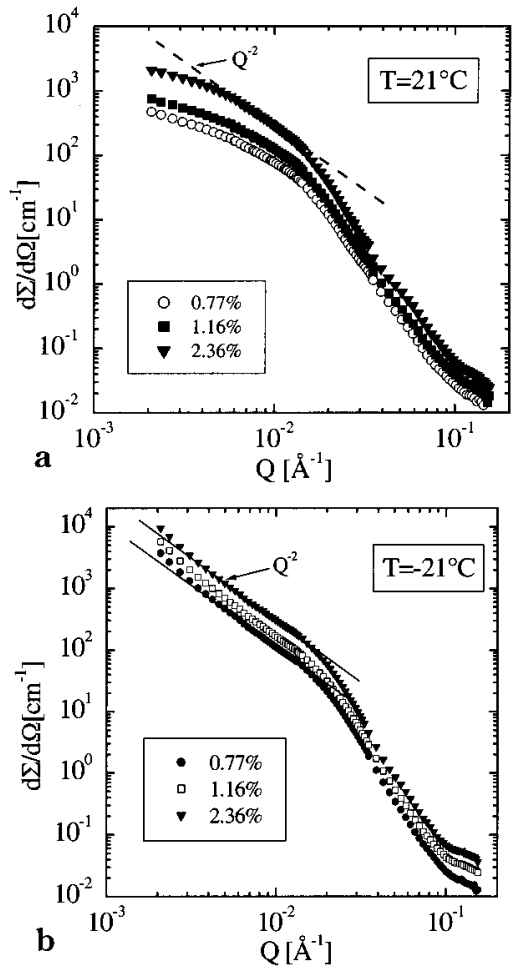

Figure 17. Scattering pattern from the PE - PEP (DVB) stars at $\mathrm{T}=+21$ (a) and $\mathrm{T}=-21^{\circ} \mathrm{C}$ (b) for different polymer volume fractions. The straight lines in part $b$ indicate the asymptotic $\mathrm{Q}^{-2}$ power law assumed at low-Q and low temperature. The same asymptote is indicated in part a by a dashed line.

Assuming that not all polymers assemble, then $\Phi_{\text {pol }}^{\text {agg }}$ and $\Phi_{\mathrm{pol}}^{\text {sol }}$ are finite with $\Phi_{\mathrm{pol}}^{\mathrm{agg}}$ exceeding $\Phi_{\mathrm{pol}}^{\mathrm{sol}}$, as the polymers are more condensed in the aggregates. Because of mass conservation the total amount of polymer stays constant and we have

$$
\Phi_{\mathrm{pol}}^{\mathrm{agg}} \Phi_{\mathrm{rod}}+\Phi_{\mathrm{pol}}^{\mathrm{sol}}\left(1-\Phi_{\mathrm{rod}}\right)=\Phi
$$

Rearrangement gives: $\Delta \Phi \equiv \Phi_{\mathrm{pol}}^{\mathrm{agg}}-\Phi_{\mathrm{pol}}^{\mathrm{sol}}=\left(\Phi-\Phi_{\mathrm{pol}}^{\mathrm{sol}}\right) /$ $\Phi_{\text {rod }}$ as represented by the thin solid lines in Figure 13 for various $\Phi_{\mathrm{pol}}^{\mathrm{sol}}$. The scattered intensity from aggregates in the forward direction, according to eq 3 is proportional to the area of the aggregates $F_{\text {rod }}$ and the coherent scattering length density $\Delta \rho_{\text {rod }}{ }^{2}$ of aggregates and the solvent. $\Delta \rho_{\text {rod }}$ is related to $\Delta \rho \equiv\left(\rho_{\mathrm{P}}-\rho_{\mathrm{S}}\right)$ according to $\Delta \rho_{\text {rod }}=\Delta \Phi \Delta \rho$. The difference $\Delta \rho \equiv\left(\rho_{\mathrm{P}}-\right.$ $\left.\rho_{\mathrm{s}}\right)$ is known from the constitution of the molecules and from tabulated values of scattering length of the atoms. One finally gets the expression:

$$
\Delta \Phi=\left\{\sqrt{\mathrm{Q} \frac{\mathrm{d} \Sigma}{\mathrm{d} \Omega}(0) /\left(\pi \mathrm{F}_{\mathrm{rod}} \Delta \rho^{2}\right)}\right\} /\left\{\sqrt{\Phi_{\mathrm{rod}}\left(1-\Phi_{\mathrm{rod}}\right)}\right\}
$$

It is plotted as the solid and dashed lines in Figure 13 for the PEB-11 and the t-PE-PEP samples at $\Phi=2 \%$ and $-30{ }^{\circ} \mathrm{C}$. The lines were calculated on the basis of the values for $\mathrm{Q}(\mathrm{d} \Sigma / \mathrm{d} \Omega)$ and $\mathrm{F}_{\text {rod }}=\pi \mathrm{a}^{2}$ from Table 3 . There is insufficient information available to evaluate from the intercepts of the curves in Figure 13 single valued parameters for the polymer volume fractions in solution and in the aggregates. In Figure 14, the polymer volume fractions $\Phi_{\mathrm{pol}}^{\mathrm{agg}}$ and $\Phi_{\mathrm{pol}}^{\mathrm{sol}}$ in the aggregates and solution have been plotted vs the volume fraction of the precipitated aggregates. F rom this figure, we recognize that depending on the aggregate volume fraction the polymer density in the aggregates could be rather low. The maximum possible volume fraction of the aggregates is 30\% and $50 \%$ for the $2 \%$ PEB- 11 and tapered samples, respectively. I n this case all polymers would be inside the aggregates with $\Phi_{\text {pol }}^{\text {sol }}$ equal to zero and $\Phi_{\mathrm{pol}}^{\mathrm{agg}}=6 \%$ and $4 \%$, respectively. If the aggregates would precipitate into smaller volume fractions, the polymer volume fractions in the aggregates and in the solvent would increase as shown in Figure 14. The observation that the scattering pattern from the $\Phi_{\text {pol }}=$ $1 \%$ and $\Phi_{\text {pol }}=2 \%$ systems (at least for PEB-11) are identical argues in favor of a low volume fraction of aggregates. Structure factor contributions relating to interaction effects between the rods are absent.

These findings confirm the notion of the previous section, namely, that the aggregates represent open objects. Both the PEB-11 and the t-PE - PEP polymers are copolymers with segments of Iinear polyethylene modulated by branching with methyl or ethyl side groups. The PE blocks have different lengths and are statistically distributed along the PEB-11 chains. Such PE blocks occur with higher probability than a statistical distribution in the PE rich segment of the t-PEPEP polymer. We may assume that the aggregation occurs as a consequence of a crystallization process of the crystallizable sections. Thus, cocrystallization of different chains may also occur. It remains to be understood, why rod structures instead of loose 3-d networks are preferred. One possibility may be a crystallization process, where in the first step PE-chain segments within one polymer start to crystallize, while the amorphous parts form a loose corona around the crystalline nucleus. Such a corona would shield the crystal against the intrusion of other chains by osmotic repulsion. The rare cocrystallization events, then, would lead to the preferential linear structures. With lower probability higher order cocrystallization occurs leading to the cross links of the loose network built by the rodlike structures. The polymer volume fraction within the rods then amounts to $4 \% \leq \Phi \leq 20 \%$ depending on the actual volume fraction filled by the aggregates (see Figure 14).

E. Aggregation Behavior of the EVA Copolymer. The EVA copolymer contains PE sections of varying length combined with the copolymerized vinyl acetate (VA). The VA comonomer is present at $9 \mathrm{wt} \%$. The temperature-dependent scattering patterns for the EVA 
Table 4. Definitions of Polymer Volume Fractions

$\Phi_{\mathrm{pol}}^{\mathrm{agg}}=$ volume fraction of the polymer in the aggregate

$\Phi_{\text {pol }}^{50 l}=$ volume fraction of the polymers in solution

$\Delta \Phi=\phi_{\text {pol }}^{\text {agg }}-\phi_{\text {pol }}^{\text {sol }}$

$\Phi_{\mathrm{A}}=$ volume fraction of the aggregate in the sample

sample (in $2 \%$ solution) are shown in Figure 15 . A distinct rise of the scattering intensity sets in al ready at temperatures around $40^{\circ} \mathrm{C}$. Particularly noteworthy is the strong increase of scattering at low Q. Since only a narrow $\mathrm{Q}$ range in the low-Q regime is covered $\mathrm{a}$ definition of a power law is difficult in that one gets exponents of more than 5. Such a high exponent cannot be interpreted in terms of fractal aggregates or fractal surfaces and most likely ori gi nates from diffuse surface scattering where the Porod exponent 4 is further increased by a smeared outer surface. ${ }^{13,14}$ If this interpretation is correct, then EVA forms large aggregates with pronounced surfaces and aggregate sizes well above several $10^{3} \AA$. It is the existence of an aggregate surface which distinguishes the EVA aggregates from those of the other materials. EVA, therefore, must devel op much more compact structures than the other aggregating polymers. This implies that the surface area available for wax nucleation is diminished. This, in turn, translates into an ateriuated activity as a wax nucleation platform relative, e.g., to the commercial diblock.

Another feature is the presence of a peak structure around $\mathrm{Q}^{*}=0.025 \AA^{-1}$. Such a peak indicates a structural correlation length of $\mathrm{R}=2 \pi / \mathrm{Q}^{*} \sim 250 \AA$. Finally, the temperature-dependent intensity loss at high Q indicates the loss of "single" polymer scattering due to an embedding of the chains into a more compact structure as well as possible sedimentation effects. Most likely both effects play a role, since a white precipitate settles over time in the sample cuvette and the large compact structures will reduce the polymer coil scattering. This lack of micelle stability at lower temperatures will compromise nucleator activity. In contrast, the prototype commercial PE-PEB diblock (and the corresponding PE-PEP along with t-PE-PEP and PEB-11) self-assembled aggregates remain in solution at $-30{ }^{\circ} \mathrm{C}$.

\section{Experimental Results and Analysis of the Crystalline and Amorphous Star Copolymers}

An aspect of star branched structures that has remained unreported upon is the influence of architecture and segment (crystalline or amorphous) placement upon the self-assembling process in solution. For example, it might be easily concluded that the placement of the crystalline segments as the corona would lead to a gelled system as a consequence of the anticipated ease of the self-assembly process involving the terminal blocks of this multi-functional polymer architecture.

A. Single Star Form F actors. We studied the single star form factors at $70^{\circ} \mathrm{C}$ as at these high temperatures self-assembly is not favored. To exclude structure factor effects, we varied the star volume fractions from $\Phi=$ $0.24 \%$ to $\Phi=2.36 \%$. F igure 16 displays the measuredcross sections for the PE - PEP (DVB) star and from the PEP-PE (DVB) star at lowest volume fraction $\Phi=$ $0.24 \%$. The SANS profile follows the typical features for star scattering with a low-Q Guinier regime, on intermediate Q strong intensity decrease, where the scattering changes from coherent to incoherent superposition of the arm scattering, to the high-Q single chain
$\rho_{\mathrm{A}}=$ scattering length density of the aggregates

$\rho_{\mathrm{B}}=$ scattering length density of solution

$\rho_{\mathrm{S}}=$ scattering length density of pure solvent

$\rho \mathrm{P}=$ scattering length density of pure polymer

Table 5. Single Star Properties from SANS

\begin{tabular}{lll}
\hline & $\begin{array}{c}\text { PE-PEP } \\
\text { (DVB) star }\end{array}$ & $\begin{array}{c}\text { PEP-PE } \\
\text { (DVB) star }\end{array}$ \\
\hline $\mathrm{d} \Sigma / \mathrm{d} \Omega / \Phi\left(\mathrm{cm}^{-1}\right)$ & 1860 & 1880 \\
$\mathrm{~V}_{\mathrm{W}, \mathrm{arm}\left(\mathrm{cm}^{3} / \mathrm{mol}^{\prime}\right)}$ & 7520 & 6050 \\
$\mathrm{R}_{\mathrm{g}, \mathrm{arm}}(\AA)$ & $56.9 \pm 0.4$ & 68 \\
$\mathrm{f}_{\mathrm{arm}}$ & 31.5 & 31.7 \\
$\mathrm{R}_{\mathrm{g}}(\AA)$ & 97.6 & 116
\end{tabular}

scattering which is well described by a $\mathrm{Q}^{-1 / v}$ power law with a Flory exponent $v=3 / 5$ for dominant excluded volume interaction.

For further discussion, we consider the cross sections for single stars

$$
\frac{\mathrm{d} \Sigma}{\mathrm{d} \Omega}(\mathrm{Q})=\left(\Delta \rho^{2} / \mathrm{N}_{\mathrm{A}}\right) \Phi \mathrm{V}_{\text {arm }} \mathrm{f}_{\text {arm }} \mathrm{P}(\mathrm{Q})
$$

where the parameters are defined as follows: $\Delta \rho^{2}$ the contrast between polymer and solvent; $\Phi=$ polymer volume fraction; $\mathrm{V}_{\text {arm }}=$ molar volume of 1 arm; $\mathrm{f}_{\text {arm }}=$ number of arms and $\mathrm{P}(\mathrm{Q})=$ the star form factor. For Gaussian stars the Debye form factor is expressed $a^{8}$

$$
\begin{aligned}
P(Q)=2\left\{u^{2}-\left[1-\exp \left(-u^{2}\right)\right]+\right. \\
\left.(f-1)\left[1-\exp \left(-u^{2}\right)\right]^{2} / 2\right\} /\left(f u^{4}\right)
\end{aligned}
$$

with $\mathrm{u}^{2}=\mathrm{Q}^{2}\left(\mathrm{R}_{\mathrm{g}}{ }^{2}\right)$ arm. The solid lines in Figure 16 display a fit of eq 7 to data obtained at the lowest star volume fraction where interaction effects are virtually absent. The fit depicts the low-Q profile as well as the initial fast decay of the scattering pattern very well. At higher $\mathrm{Q}$, as a consequence of the assumption of Gaussian chain statistics, severe deviations are evident. The obtained fit parameters are listed in Table 5.

For the PE -PEP (DVB) star, SANS yields a molecular volume of $2.36 \times 10^{5} \mathrm{~cm}^{3} / \mathrm{mol}$ leading to $\mathrm{M}_{\mathrm{w}}=2 \times$ $10^{5} \mathrm{~g} / \mathrm{mol}$ in very close agreement with the characterization result of $2.05 \times 10^{5}$. The radius of gyration for one arm was obtained from $\left.\mathrm{R}_{\mathrm{g}}{ }^{2}\right|_{\text {star }}=\left.(3 \mathrm{f}-2 / \mathrm{f}) \mathrm{R}_{\mathrm{g}}{ }^{2}\right|_{\mathrm{arm}}$. Its value of $57 \AA$ is significantly larger than the unperturbed radius which for the arm under consideration would be $R_{\mathrm{g}}$ arm $_{2} \simeq 30 \AA$. Obviously the star is swollen and the arms are stretched. For the other star (PEPPE (DVB)), the agreement between the SANS characterization and the sample characterization by light scattering is also favorable. While the absolute cross section points to a nearly identical star molar volume of $2.39 \times 10^{5} \mathrm{~cm}^{3} / \mathrm{mol}\left(\mathrm{M}_{\mathrm{w}} \cong 2.0 \times 10^{5} \mathrm{~g} / \mathrm{mol}\right)$ the characterization result was $M_{w} \cong 2.06 \times 10^{5} \mathrm{~g} / \mathrm{mol}$. The SANS results in a larger $R_{g}=116 \AA$ yielding also a larger $R_{g}^{a r m}=68 \AA$. This may be a reflection of differences in the DVB core geometry. This can be inferred to exist from a kinetic evaluation of the crossover reaction involving the dienyl headgroups and DVB. ${ }^{9}$

B. Aggregation Behavior of the Block Copolymer Stars. We first consider the PE -PEP (DVB)-star (Table 2) where the crystallizable PE blocks are placed at the rim of the star corona. Figure 17 presents the SANS results at +21 and $-21^{\circ} \mathrm{C}$ for three different star volume fractions. In comparison to the single star form factors displayed in Figure 16, we now observe an 


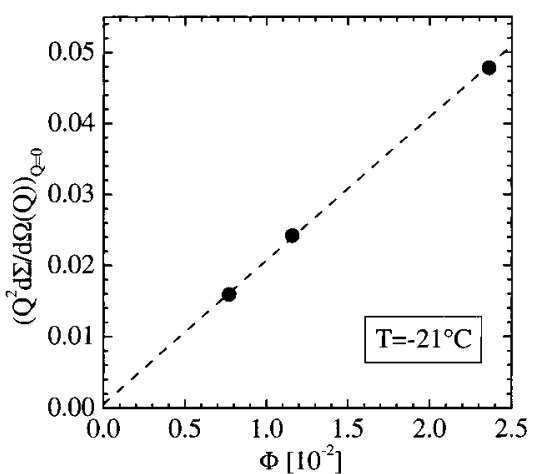

Figure 18. F orward scattering from the Guinier approximation for the PE-PEP (DVB) star at $\mathrm{T}=-21^{\circ} \mathrm{C}$ as a function of polymer volume fraction.

Table 6. Molecular Parameters for the Self-Assembled PE-PE P(DVB) Star-Based Disks at $-21^{\circ} \mathrm{C}$

\begin{tabular}{|c|c|c|c|c|}
\hline$\Phi(\%)$ & $d_{\text {eff }}(\AA)$ & $\begin{array}{c}\mathrm{Q}^{2} \mathrm{~d} \Sigma / \mathrm{dQ}(\mathrm{Q}=0) \\
\left(10^{14} \mathrm{~cm}^{-3}\right)\end{array}$ & $\begin{array}{c}\Delta \rho_{\text {plate }} \\
\left(10^{10} \mathrm{~cm}^{-2}\right)\end{array}$ & $\Phi_{\text {pol }}^{\text {plate }}$ \\
\hline $\begin{array}{l}0.77 \\
1.16\end{array}$ & $\begin{array}{l}136.2 \pm 0.8 \\
140.8 \pm 0.7\end{array}$ & $\begin{array}{l}1.59 \pm 0.02 \\
2.42 \pm 0.02\end{array}$ & $\begin{array}{l}3.51 \\
3.45\end{array}$ & $\begin{array}{l}0.51 \\
0.50\end{array}$ \\
\hline 2.36 & $147.6 \pm 0.8$ & $4.78 \pm 0.02$ & 3.17 & 0.46 \\
\hline
\end{tabular}

intensity which is steadily increasing toward low Q indicating the presence of aggregation processes. In particular at $\mathrm{T}=-21^{\circ} \mathrm{C}$ a well defined $\mathrm{Q}^{-2}$ power law is assumed which is characteristic for the scattering from plates. In the Guinier approximation the corresponding structure factor has the form ${ }^{2}$

$$
\frac{\mathrm{d} \Sigma}{\mathrm{d} \Omega}=\Phi^{\text {plate }} \Delta \rho_{\text {plate }}{ }^{2} 2 \pi \mathrm{d}_{\text {eff }} \exp \left[-\mathrm{Q}^{2} \mathrm{~d}_{\text {eff }}^{2} / 12\right] / \mathrm{Q}^{2}
$$

where $\Phi^{\text {Plate }}$ is the volume fraction of the plates, $\Delta \rho_{\text {plate }}$ the corresponding scattering length density contrast, and $d_{\text {eff }}$ the effective thickness of the plates.

Figure 18 shows the forward scattering of the PEPEP(DVB) as a function of the polymer volume fraction as evaluated from a modified Guinier plot $\ln ((\mathrm{d} \Sigma / \mathrm{d} \Omega)$ $\left.\mathrm{Q}^{2}\right)$ vs $\mathrm{Q}^{2}$. The observed linearity and the zero intercepts demonstrate that all of the star molecules at each concentration have participated in the self-assembly event.

The platelets contain crystalline as well as amorphous star sections. The platelets with the effective thickness $d_{\text {eff }}$ contain a certain fraction of d-decane, which may be obtained from the absolute intensities. From eq 8, we have

$$
\Phi_{\mathrm{pol}}^{\text {Plate }}=\frac{\mathrm{Q}^{2}(\mathrm{~d} \Sigma / \mathrm{d} \Omega)(\mathrm{Q}=0)}{\Phi_{\mathrm{pol}} 2 \pi \mathrm{d}_{\mathrm{eff}}\left(\rho_{\mathrm{pol}}-\rho_{\mathrm{sol}}\right)^{2}}
$$

Inserting the experimental results from Table 6 and using $\rho_{\text {pol }}-\rho_{\text {sol }}=6.89 \times 10^{10} \mathrm{~cm}^{-2}$, we arrive at a polymer volume fraction $\Phi_{\text {pol }}^{\text {plate }}$ within the plates of about $50 \%$ (see Table 6).

With the firm establishment of plate structures at low temperatures we may interpret the data at $21{ }^{\circ} \mathrm{C}$ (Figure 17a) as due to plates of a size not yet large enough to display the asymptotic $\mathrm{Q}^{-2}$ law over a larger $Q$ range. In particular the data at $\Phi_{\text {pol }}=2.36 \%$ display an intermediate $\mathrm{Q}^{-2}$ regime (see dashed line) which is followed by Guinier behavior toward the lowest Q values. We may conclude that, at $\mathrm{T}=21^{\circ} \mathrm{C}$, the stars al ready assemble into plates with sizes on the order of $\sim 100 \mathrm{~nm}$.

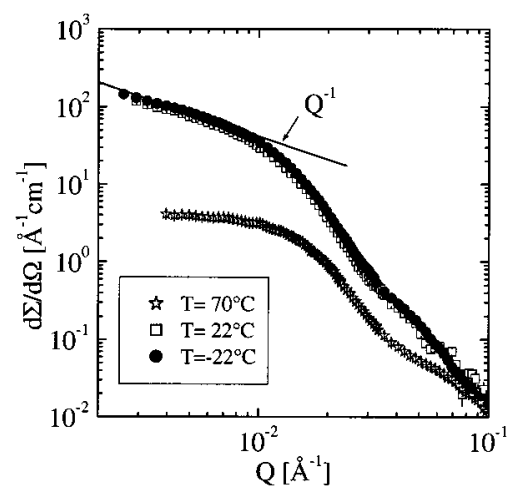

Figure 19. SANS profiles for the PEP-PE (DVB) star (crystallizable blocks in the star center) at $\Phi_{\mathrm{pol}}=0.24 \%$ for three different temperatures. Note the $\mathrm{Q}^{-1}$ power laws assumed by the data at lower temperatures (solid line).

We now turn to the PEP-PE (DVB) star, where the crystallizable blocks are placed in the star center. Figure 19 presents the temperature-dependent SANS results for $\Phi_{\mathrm{pol}}=0.24 \%$. Again we observe a strong intensity increase with decreasing temperature. However, other than for the star with the crystallizable parts at the corona rim we now observe (i) an asymptotic $\mathrm{Q}^{-1}$ law at low $\mathrm{Q}$ which (ii) does not change bel ow room temperature. Obviously, these stars aggregate into rods or needl es which already at room-temperature encompass all stars. They are of a length much larger than $\sim 100$ $\mathrm{nm}$ such that within the observation window of SANS asymptotic laws are observed. For a small fraction of rods and a complete aggregation of the polymer eq 3 transforms into

$$
\frac{\mathrm{d} \Sigma}{\mathrm{d} \Omega} \mathrm{Q}=\Phi_{\mathrm{rod}} \Delta \rho_{\mathrm{Pol}}{ }^{2}\left(\Phi_{\mathrm{pol}}^{\mathrm{rod}}\right)^{2} \mathrm{a}^{2} \pi^{2} \exp \left[-\mathrm{Q}^{2} \mathrm{a}^{2} / 4\right]
$$

where $\Phi_{\text {rod }}$ is the volume fraction of the rods and $\Phi_{\text {pol }}^{\text {rod }}$ is the volume fraction of the polymers within the rods. A 1-d Guinier plot $\left(\ln (\mathrm{Q}(\mathrm{d} \Sigma / / \mathrm{d} \Omega))\right.$ vs $\left.\mathrm{Q}^{2}\right)$ leads to the rod radius $\mathrm{a}=(146 \pm 1) \AA$ and the forward scattering $\mathrm{Q}[\mathrm{d} \Sigma /$ $\mathrm{d} \Omega](0)=0.584 \times 10^{8} \mathrm{~cm}^{-2}$. Following an analogous evaluation as that based in eq 9 , we find for the overall polymer volume fraction within the $\operatorname{rod} \Phi_{\text {pol }}^{\text {rod }}=0.24$. Finally, relating the rod radius with the radius of gyration of the single star $\left(R_{g}=116 \AA\right.$, see Table 5$)$, $\mathrm{R}_{\mathrm{g}}^{\text {rod }}=\mathrm{a} \sqrt{\frac{3}{4}}=126 \AA$, our results suggest that the rods consist of a 1-d array of single stars. The structure is displayed as a cartoon in Figure 20. Obviously a very stiff entropy penalty forbids the association in the form of plates.

Comparing the results from the stars with amorphous sections at the rim or the center of the corona we note that the placement of the crystalline blocks controls the identity of self-assembled structures. PE blocks at the corona rim are little restricted and crystallize in their normal plate structure dictated by the crystallization enthalpy. On the other hand, if the PE blocks are at the star center, then the outside corona strongly shields the crystallization event from interaction with other stars. The rare cases, where a joint crystallization occurs, leads to 1-d growth assembling stars such as pearls on a necklace. Thus, the amorphous shell to a large extend screens cocrystallization events between different stars.

We may assume that the same mechanism is also behind the surprising rod formation of the PEB-11 self- 


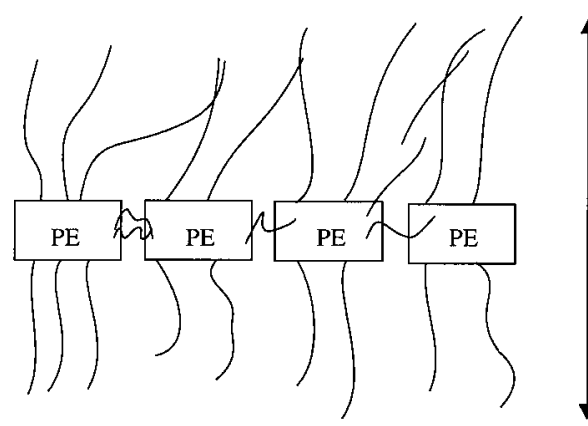

$290 \AA$

Figure 20. Cartoon of the rod structures formed by the PEPPE (DVB) stars.

assembly. There initial crystallization events may occur within a given chain pushing the amorphous sections to the outside. This creates a similar situation as realized by the stars with an amorphous outer corona. Cocrystallization events between different chains are screened and one-dimensional growth is promoted.

\section{Summary and Conclusions}

We have investigated the self-assembly of copolymers with crystalline and amorphous sections in solutions. Thereby, both the chain topography as well as the polymer architecture was varied.

(i) Star Polymers. A key observation leading to a qualitative understanding of the observed structures concerns the self-assembly of stars with crystallizable PE blocks either at the star rim or in the star center. When PE is at the core of the star the self-assembled micelles take the form of quasi-one-dimensional needle shaped structures. Conversely, platelike structures emerge when the star core contains the amorphous PEP. This observation appears to indicate that the screening effects of the amorphous corona is detrimental for the assumed structure.

(ii) Random Copolymers. In dilute solution in decane upon cooling the random copolymer PEB-11 forms 1-dimensional rodl ike structures. This aggregate architecture was a surprise in view of the expectation of loosely connected 3-d networks. In view of the star results we may conjecture that the formation of the rod structures arises from the presence of amorphous polymer loops and strands attached to the initial crystallites. The PEB-11 sample does not commence selfassembly until about $0{ }^{\circ} \mathrm{C}$. We expect that the selfassembly temperature is tunable by the ethyl branch content until crystallinity vanishes (this occurs for PE B14 to -15$)$.

(iii) Tapered Copolymers. The tapered t-PE-PEP sample yields signs of self-assembly at about $50{ }^{\circ} \mathrm{C}$, which is reminiscent of the behavior of the discrete diblock structure. ${ }^{3}$ The high self-assembly temperatures may be related to the presence of longer ethylene segments, which are clustered at one end of the chain. The self-assembly process continues to progress as temperature decreases. The structures formed start as fractal networks which toward lower temperatures display some preferred 1-d rodlike behavior. However, compared to the PEB-11 the rod sizes are significantly smaller. At large scales, the fractal network character dominates. At $-30{ }^{\circ} \mathrm{C}$ these networks become 3-d porous objects which remain perfectly soluble. On the other hand the t-PE -PEP sample failed to show any capacity for platelet formation, which is the hallmark of the corresponding discrete commercial diblocks.

The feature of an extended self-assembly temperature range (ca. +50 to $-20^{\circ} \mathrm{C}$ ) for the tapered material and bel ow $0{ }^{\circ} \mathrm{C}$ for the PEB-11 implies that these materials may be suitable for cocrystallization with a range of wax forming alkanes.

(iv) Commercial Wax Crystal Modifier. Finally, the commercial EVA wax crystal modifiers aggregate into compact structures with a diffuse surface. Furthermore, the EVA aggregates precipitate from solution at low temperatures. For wax crystal modification this implies an efficiency penality which adds to the significantly smaller surface offered for nucleation by the compact structures. The linear and star shaped poly(co-olefins) along with the commercial diblock do not share that problem.

\section{References and Notes}

(1) Richter, D.; Schneiders, D.; Monkenbusch, M.; Willner, L.; Fetters, L. J .; Huang, J. S.; Lin, M.; Mortenson, K.; Farago, B. Macromolecules 1997, 30, 1053.

(2) Monkenbusch, M.: Schneiders D.: Richter, D : Farago, B : Fetters L. J .; Huang, J . Nuovo Cimento 1994, 16, 747.

(3) Leube, W.; Monkenbusch, M.; Schneiders, D.; Richter, D.; Adamson, D.; Fetters, L. J .; Dounis, P.; Lovegrove, R. Energy Fuels 2000, 14, 419

(4) Morton, M.; Fetters, L. J . Rubber Chem. Technol. 1975, 48, 359.

(5) Bi, L. K.; Fetters, L. J . Macromolecules 1976, 9, 732.

(6) Higgins, J . S.; Benoit, H. Polymers and Neutron Scattering; Clarendon Press: Oxford, England, 1994.

(7) Pennisi, R.; Fetters, L. J . Macromol ecules 1988, 21, 1094

(8) Grest, G.; Fetters, L. J .; Huang, J . S.; Richter, D. Adv. Chem. Phys. 1996, 94, 67.

(9) Lindner, P. J. Appl. Crystallogr. 2000, 33, 807.

(10) Avnier, D.; Biham, O.; Lidar, D.; Malcai, O. Science 1998 39, 279.

(11) Beaucage, G. J . Appl. Crystallogr. 1995, 28, 717

(12) Young, R. N.; Fetters, L. J . Macromolecules 1978, 11, 899.

(13) Ruland, W. Macromolecules 1987, 20, 87.

(14) Hashimoto T. Structure of Polymer Blends. In Materials Science and Technology; Cohn, R. W., Haasen, P., Kramer, E. J., Eds.; In Structureand Properties of Polymers; Thomas, E. L., Ed.; VCE: Weinheim, Germany, 1991; Vol. 12, p 251. Müller, G.; Schwahn, D.; Springer, T. Phys. Rev. E 1997, 55, 7267.

MA0111818 